\title{
LA EVOLUCIÓN DEL CONCEPTO DE ESTRATEGIA DE MARKETING INTERNACIONAL Y SU APLICACIÓN AL CASO ESPAÑOL DE MODA HOGAR
}

\section{THE EVOLUTION OF THE EXPORT MARKETING STRATEGY CONCEPT AND ITS APPLICATION TO THE HOME DECORATION SECTOR IN SPAIN}

Fernando González-Ferriz (Universidad Isabel I de Castilla) ${ }^{1}$

Javier Sánchez-García (Universidad Jaume I) ${ }^{2}$

Fernando J. Garrigos-Simon (Universitat Politècnica de València) ${ }^{3}$

\section{Resumen:}

La estrategia de marketing internacional es una de las variables más importantes entre los factores que determinan el resultado de una empresa exportadora. Este trabajo pretende profundizar en el tema mediante el análisis del concepto teórico de marketing y su evolución a lo largo de las últimas décadas, resultando en un cambio de tendencia que avanza desde un tipo de marketing centrado en el producto a otro centrado en los servicios. De esta forma, se van a considerar aspectos como el marketing relacional, la responsabilidad social corporativa o la incorporación de las nuevas tecnologías al proceso operativo, que serán analizados desde un trasfondo teórico basado en el punto de vista de los recursos de la empresa (RBV) y la teoría de las capacidades dinámicas. Por otra parte, el análisis cuantitativo de las políticas de marketing estratégico y operativo en el sector de la moda hogar en España mostrará, gracias a los testimonios de los propios directivos, la forma en la que las empresas llevan a cabo sus estrategias de marketing internacional, identificando los puntos fuertes y débiles para contribuir a la mejora de la competitividad empresarial del sector.

Palabras Clave: Gestión empresarial; Marketing internacional; Estrategia; Concepto de Marketing; Dirección de empresas.

Códigos JEL:M16, M31.

\section{Abstract:}

The export marketing strategy is one of the most important variables among the determinants of the export performance in international companies. This paper pretends to focus on this subject through the analysis of the theoretical concept of marketing and its evolution throughout the last decades, resulting in a change of tendency, which moves from a product based kind of marketing to another based on services. In this context, aspects like relational marketing, corporate social responsibility or the incorporation of new technologies to the operational process will be considered and analyzed from a consistent theoretical background based on the Resource Based View (RBV) and the theory of dynamic capabilities. Additionally, the quantitative analysis of the strategic and operational marketing policies in the home decoration industry in Spain will show, thanks to the testimony of the managers themselves, the way companies carry out their international marketing strategies, giving us

\footnotetext{
${ }^{1}$ fernando.gonzalez.ferriz@ui1.es, Universidad Isabel I de Castilla.

2jsanchez@uji.es, Universidad Jaume I.

33fgarrigos@doe.upv.es, Universitat Politècnica de València.

Recibido 1 de septiembre de 2020. Aceptado 29 de noviembre de 2020.
} 
the opportunity to identify their strengths and weaknesses in order to contribute to the improvement of the competitive advantage in the industry.

Keywords: Business management; International marketing; Strategy; Marketing concept; Business Management

JEL Codes:M16, M31.

\section{INTRODUCCIÓN}

Es evidente que las exportaciones están jugando un papel fundamental en el desarrollo de la economía española en las últimas décadas y no han dejado de crecer desde principios de siglo, con la única excepción de dos años en la crisis de 2008 y más recientemente en 2020 debido a los efectos de la COVID-19 (Base de datos Estacom, Instituto Español de Comercio Exterior; ICEX).Por ello, el análisis del rendimiento de las empresas exportadoras se ha presentado tradicionalmente como un tema de investigación que suscita gran interés, tanto en el sector empresarial como en el académico. Entre los factores que determinan ese rendimiento juega un papel fundamental la estrategia de marketing de la compañía, que suele actuar como una variable intermedia, afectada por aspectos como el entorno en mercados nacionales e internacionales, las características de la gestión empresarial, y el resto de capacidades y activos intangibles desarrollados por cada empresa.

Aunque el marketing es una disciplina que goza de gran salud, cabe destacar que ha sufrido grandes transformaciones, especialmente en los últimos años. Al tratarse de una ciencia con un importante componente social ha debido adaptarse al nuevo entorno económico, de gran complejidad, altamente globalizado y afectado por cambios constantes que deben ser analizados e interpretados. En este nuevo entorno, las empresas deben ser más productivas, intentando minimizar sus costes y maximizar sus beneficios, resultando un claro beneficiado, el consumidor, que cada vez encuentra mayor cantidad de productos a precios menores (Czinkota y Ronkainen, 2011).

Sin embargo, es importante destacar que esa evolución de la disciplina no implica desechar las teorías tradicionales, sino que más bien se han incorporado a ellas nuevos elementos para ofrecer un marco teórico mucho más complejo y avanzado. En este sentido, si partimos de las políticas de marketing mix tradicionales, la evolución hacia una sociedad basada en los servicios ha supuesto la incorporación de nuevos instrumentos como la relación con el cliente o la incorporación de las nuevas tecnologías a esta nueva era del marketing. En este contexto, autores como Kotler $(2010,2016)$ describen la evolución de las políticas de marketing en una serie de etapas (desde la 1.0 hasta la 5.0) e incorporan nuevos elementos a las famosas 4Ps que todavía siguen en vigor, y son de gran utilidad para el desarrollo de esas nuevas estrategias de marketing internacional.

El presente estudio pretende analizar este nuevo contexto en el sector de la moda hogar española, con el objetivo de determinar el grado en que sus empresas exportadoras adaptan sus decisiones de marketing, tanto estratégico como operativo, a las nuevas políticas que vienen determinadas por aspectos tan fundamentales como la creación de relaciones a largo plazo con sus clientes mediante su fidelización, o la incorporación de las nuevas tecnologías a procesos como la investigación de mercados, la promoción y venta de sus productos a través de Internet o la mejora de la comunicación interna y externa de la empresa.

El análisis cuantitativo de los datos aportados por los directivos de una muestra representativa de empresas exportadoras en el sector indicado, nos va a ayudar, por tanto, a 
comprender mucho mejor la forma en la que se lleva a cabo hoy en día la planificación y la ejecución de las políticas de marketing internacional, así como las fortalezas y debilidades que presentan dichas estrategias.

Cabe destacar que el sector de la moda, y más concretamente el de moda hogar, presentan determinadas peculiaridades que deben ser tenidas cuentas a la hora de analizar sus estrategias de internacionalización. En lo que respecta al tamaño de las empresas, la mayor parte de ellas son pymes de tamaño medio y carácter familiar, con una mayor concentración geográfica en las zonas del levante español (Comunidad Valenciana y Cataluña fundamentalmente). Además, en lo que a cifras de exportación se refiere, el sector ha visto doblar sus ventas internacionales desde principios de siglo, considerándose como principales destinos nuestros socios europeos y los países árabes con los que tradicionalmente ha existido una buena relación comercial.

\section{MARCO TEÓRICO}

En lo que respecta a los principales temas de investigación en la disciplina del marketing, encontramos distintos artículos (Lehmann, 2005; Yadav, 2010; Haji-Basri, 2012; Saleh, 2016)que nos ayudarán a conocer tanto el punto de partida como la evolución de las principales áreas del marketing, agrupando los contenidos en torno a 4 grandes grupos: entorno de marketing, funciones de marketing (incluye conceptos como políticas de marketing mix, planificación y estrategia, marketing electrónico o gestión del retail), aplicación del marketing a distintos sectores, o investigación de marketing. Se observa la importancia de los estudios en torno al comportamiento del consumidor, así como la evolución al alza del área de emarketing y el gran peso que siguen ejerciendo las políticas de marketing mix. También destacan los estudios sobre gestión del marketing y el retail, y aquellos centrados en el marketing relacional (relación con el cliente, satisfacción del cliente, creación de valor, servicios y calidad del servicio, responsabilidad social corporativa...).

Otros autores (Oh et al., 2004; Line y Runyan, 2012) determinaron las principales áreas de investigación en lo que a marketing se refiere en los sectores de servicios y hostelería, destacando la siguiente clasificación por áreas, llevada a cabo por Line y Runyan: comportamiento del consumidor (28,1 por cien), planificación de la gestión y estrategia (19,7 por cien), satisfacción y comportamiento de los trabajadores (12,8 por cien), características del consumidor (8,8 por cien), marketing electrónico (8,8 por cien) y relaciones públicas (7,3 por cien). De ello se desprende que, a pesar de la similitud en las áreas investigadas con respecto a un entorno más genérico, las empresas de este sector prestan una especial atención a las percepciones del consumidor y de los empleados, así como a las relaciones públicas en detrimento de los medios de promoción tradicionales.

\subsection{Del marketing de los productos al marketing de los servicios.}

Las primeras investigaciones en marketing comienzan con la llamada "escuela funcional” que se caracteriza por prestar una especial atención al intercambio y la distribución de materias primas y productos manufacturados. Los bienes se producían en masa y era necesario facilitar el intercambio de productos a través de instituciones especializadas en marketing. Sin embargo, a partir de 1950 surge un nuevo enfoque conocido como la "escuela de gestión del marketing", que se centraba en un proceso de toma de decisiones para gestionar de forma efectiva las acciones de marketing, considerando al cliente como el centro de la estrategia. Es a partir de los 60 cuando algunos autores definen un nuevo enfoque basado en la satisfacción del cliente mediante el estudio del mercado y la toma de decisiones a través de las políticas de "marketing mix", introduciendo el concepto de las 4Ps (Producto, Precio, 
Promoción y Distribución) que sigue en vigor en la actualidad. Posteriormente, a partir de los 80, surgen nuevas corrientes basadas en la orientación al mercado y la gestión de cuestiones como la cadena de valor, la calidad, los recursos, las relaciones o las redes, que contribuyen al desarrollo de una "subdisciplina" para dar explicación al marketing de los servicios (ver tabla 1). Mientras unos sostienen la aparición de un paradigma alternativo a la teoría del "intercambio de bienes", otros defienden este hecho como la evolución de la ciencia de la comercialización hacia un enfoque mucho más especializado (Vargo y Lusch, 2004, 2016).

\section{TABLA 1. EVOLUCIÓN DEL MARKETING DE LOS PRODUCTOS AL DE LOS SERVICIOS}

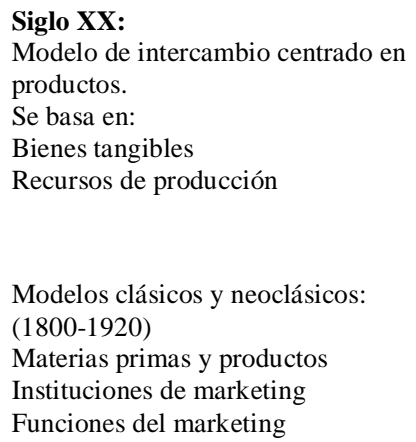

Evolución de un modelo
basado en productos
(tangibles) a un modelo
basado en servicios
(intangibles)

Escuela de gestión del marketing: (1950-2000)

Orientación al cliente

Valor determinado por el mercado

El mktg proporciona un resultado óptimo
Siglo XXI:

Modelo de intercambio centrado en servicios.

Se basa en:

Bienes intangibles

Relaciones

Procesos de intercambio

Marketing social (2000-actualidad):

Orientación al mercado

Marketing de los servicios

Gestión de la calidad

Gestión de las redes (mktg relacional)

Gestión de los recursos (RBV)

Fuente: Adaptación de Vargo y Lusch 2004, 2016.

\section{A. La orientación al mercado}

Este enfoque determina que la orientación al mercado de cualquier negocio es un factor determinante a la hora de evaluar su rendimiento, independientemente de las turbulencias en el mercado, la intensidad competitiva o los cambios tecnológicos del entorno (Narver y Slater, 1990; Jaworski y Kohli, 1993). Esa orientación al mercado implicará la creación de un mayor valor para el cliente, que contribuirá a una ventaja competitiva sostenible, y ésta a su vez repercutirá en un mejor resultado empresarial.

El concepto de orientación al mercado ha evolucionado a lo largo de las últimas décadas y muchos autores han destacado su contribución desde el punto de vista estratégico a la mejora del rendimiento empresarial (Guo, 2002; Pena et al., 2012; Chad, 2014; Balodi, 2014; Shafei y Zohdi, 2014). Además, esta corriente no solo contribuye a fomentar la generación e intercambio de información, sino que también desarrolla una cultura basada en la comunicación dentro de la empresa, que revertirá en la mejora de las capacidades relacionales como activos intangibles de la empresa (Shafei y Zohdi, 2014; Wei et al., 2014; Devece et al., 2017). Finalmente, la teoría de la orientación al mercado supone el punto de partida para el desarrollo de otros enfoques como el marketing relacional, la perspectiva basada en los recursos o la teoría de las capacidades dinámicas, que son analizadas más adelante.

\section{B. Marketing relacional y análisis de redes.}

El marketing relacional se basa en fomentar, mantener y desarrollar las relaciones a largo plazo, fundamentalmente con los clientes. Estas relaciones, además, pueden ser con otros miembros y organizaciones (proveedores, miembros del canal de distribución...), y se convertirán en redes cuando sean más frecuentes y complejas. Para muchos autores (Berry, 1983; Gummesson, 1994a, 1994b, 2002; Sheth y Parvatiyar, 2000; Payne y Frow, 2005) se 
trata de un enfoque independiente que se puede aplicar tanto a los bienes de consumo como a los servicios y a las interacciones B2B (business to business).

Otro aspecto importante del marketing relacional es la gestión de la relación con el cliente, que se define como la aplicación de la tecnología para obtener y analizar la información que nos proporcionan los clientes con el fin de obtener una relación a largo plazo que sea positiva para ambas partes (Berry, 1983; Gronroos, 1990; Morgan y Hunt, 1994; Vivek et al., 2012). Según Payne y Frow (2005, 2008, 2017) el concepto de CRM ha evolucionado hasta el punto de implementar soluciones integradas en la empresa con el objetivo de gestionar de la forma más eficiente posible las relaciones con el cliente y generar valor para el accionista. Por último, destacar que muchos han sido los académicos que han considerado este enfoque del marketing relacional en sus investigaciones, proporcionando a las políticas tradicionales de marketing mix un enfoque mucho más efectivo (Karelakis et al., 2008; Frow y Payne, 2009; Lages et al., 2009; Ricci y Trionfetti, 2012; Chugan y Singh, 2014; Payne y Frow 2017).

Por otra parte, la evolución desde una etapa inicial basada en transacciones individuales hasta un esquema basado en aspectos como las operaciones recurrentes, las alianzas estratégicas o los partenariados, ha definido la esencia de la teoría de la creación de redes, considerando a éstas como activos intangibles de la empresa (firm capabilities), algo que nos acerca al enfoque basado en los recursos (Resource Based View-RBV).

\section{El enfoque basado en los recursos (RBV) y la teoría de las capacidades dinámicas.}

La teoría basada en los recursos de la empresa (RBV) es uno de los enfoques con mayor aceptación en el campo de la gestión empresarial y la internacionalización. En este sentido, sus defensores mantienen que todas las empresas desarrollan una serie de recursos tangibles e intangibles, y son éstos precisamente los que determinan la ventaja competitiva de cada compañía (Penrose, 1959; Wernerfelt, 1984, 1995; Prahalad y Hamel, 1990; Barney, 1991; Nelson, 1991; Teece, Pisano y Shuen, 1997; Katsikeas et al., 2000). Además, será de gran importancia la organización interna de las empresas y las estrategias que van a implementar, ya que la forma de explotar esos recursos y capacidades las hace únicas y va a ser lo que determine la mayor o menor competitividad de cada empresa (Conner, 1991). Van a ser necesarios activos tales como el conocimiento, los avances tecnológicos, las relaciones con los clientes, el valor de la marca o la dirección de la empresa para ejecutar la estrategia de marketing (Srivastava et al., 2001; Kozlenkova et al., 2014; Barney y Hesterly, 2015). Cuando una empresa ha conseguido desarrollar una serie de recursos que otros no tienen y no pueden imitar, desarrolla una ventaja competitiva que le proporciona mejores resultados y le diferencia de sus competidores (Day, 1994; Hunt y Morgan, 1995; Fahey, 1999; Hunt, 2000; Barney y Hesterly, 2015).

No obstante, el enfoque basado en los recursos derivó hacia la teoría de las capacidades dinámicas en el momento en el que se consideraron los cambios en el entorno de las empresas. En este sentido, autores como Teece et al. (1997), Desarbo et al. (2005), Song et al. (2008), Murray et al. (2011), o Parnell et al. (2015), consideraron que las estrategias empresariales no permanecen constantes en el tiempo, sino que van cambiando en función del mercado y de las necesidades de los consumidores. Una estrategia, por tanto, puede ser acertada para una determinada empresa en un momento concreto, y no ser adecuada en circunstancias distintas o para otro tipo de compañía. La forma en la que se gestiona cada empresa y la manera de adaptarse a los cambios del mercado, va a determinar la ventaja competitiva y el resultado final de cada actividad. Entran en juego, por tanto, aspectos tan importantes como la orientación al mercado, la relación con el cliente, la gestión del conocimiento o la incorporación de las nuevas tecnologías a las políticas de marketing, que 
van a ser fundamentales en los procesos de internacionalización. En este sentido, no solo es importante la propiedad de los recursos, sino también la adquisición del conocimiento necesario para desarrollar nuevas capacidades, llevando incluso a un grupo de investigadores a considerar un nuevo enfoque conocido como la teoría basada en el conocimiento que presta una especial atención a ese tipo de recursos intangibles (Ruzzier et al., 2006).

\section{Otros enfoques.}

De forma paralela a la evolución de las principales corrientes en la teoría del marketing, han ido surgiendo una serie de tendencias que están ganando peso en los últimos años, y, aunque, en lo que a investigación se refiere, todavía queda mucho por hacer en alguno de estos campos, merece la pena destacar los aspectos más importantes.

El cambio de paradigma en la evolución del marketing desde el producto hasta los servicios, ha llevado al mundo empresarial hacia una nueva etapa basada en la economía de las experiencias, de forma que el valor añadido no se limita solo a la fabricación de bienes o la prestación de servicios, sino también a las experiencias que éstos proporcionan (Pine y Gilmore, 1999). En este contexto, Schmitt (2000, 2014), considerado como uno de los pioneros en este campo, proponía que las campañas de marketing de las empresas más grandes debían dirigirse hacia acciones que "estimularan los sentidos de los consumidores, conmovieran sus corazones y estimularan sus mentes”, ya que la calidad, los beneficios del producto y la imagen de marca se dan por hechos. Y defendía esta teoría en base a tres tendencias del marketing moderno: la omnipresencia de la tecnología de la información, la supremacía de la marca y la ubicuidad de la comunicación y el entretenimiento. Mientras que el marketing tradicional considera al consumidor final como un ser racional que toma sus decisiones en base a las características y beneficios que le ofrece el producto, el marketing experiencial se centra en un ser racional y emocional, que pretende disfrutar de experiencias agradables, incluso durante el proceso de compra (Qader y Omar, 2013), cobrando especial importancia la fidelización de un cliente satisfecho y su identificación con la filosofía de la marca (Atilgan et al., 2005; Brakus et al., 2009).

Si hay un campo que va a seguir evolucionando en los próximos años, ése va a ser sin duda el del marketing electrónico. Aunque no es un enfoque nuevo en lo que se refiere a prácticas comerciales y su aplicación a la gestión de la empresa, el marketing en internet debe responder a una serie de retos como son: el empoderamiento del cliente, las nuevas formas de comunicación, las operaciones globales, los horarios 24/7 o la dificultad para mantener e incrementar la ventaja competitiva en la empresa (Constantinides, 2014). Además, la incorporación de las nuevas tecnologías a la estrategia de marketing es de gran importancia porque proporciona tanto un modo de incrementar la promoción y las ventas, como un canal de distribución en algunos casos (Ngai, 2003; El-Gohari, 2010; Mathews et al., 2016). Otros autores (Kaplan, 2012; Saravanakumar y Sugantha Lakshmi, 2012) incluso identifican el marketing llevado a cabo a través de dispositivos móviles como una tendencia a seguir de cerca. Por último, es importante destacar que algunos académicos (Prasad et al., 2001; Song et al., 2008; Morgan y Katsikeas, 2012; Parnell et al., 2015; Mathews et al., 2016) han contemplado la incorporación de las nuevas tecnologías al marketing desde el punto de vista de los recursos, considerando éstas como activos intangibles que permiten mejorar el rendimiento de las empresas, sobre todo en el caso de las pymes donde el desarrollo de redes internacionales de contactos no es tan evidente.

La evolución del marketing como ciencia y la incorporación de nuevos elementos a las políticas tradicionales de marketing mix, se han descrito en forma de etapas por autores como Kotler (2010, 2016). Así pues, evolucionamos de un Marketing 1.0 centrado en obtener un producto al mínimo coste posible hasta un Marketing 2.0 que introduce conceptos como la segmentación, para operar con targets específicos en una era centrada en torno al cliente. A 
partir de aquí, con el marketing 3.0, el cliente es tratado no solo como un consumidor, sino como un ser humano con valores y emociones, coincidiendo con el enfoque de marketing basado en experiencias. Una etapa adicional definida por Kotler et al. (2016) nos aproxima a un marketing 4.0 con consumidores que se involucran todavía más en el proceso de decisión de la empresa, (existe una cierta interacción de ambas partes) y destacan aspectos como el valor de la marca, la reputación y el compromiso con el entorno que se manifiesta a través de las políticas de Responsabilidad Social Corporativa, una tendencia que es analizada también desde el punto de vista de los recursos e incorporada cada vez a un mayor número de modelos económicos y políticas de marketing (Friedman, 2007; Orlitzky et al., 2011; Chen et al., 2016; Ali et al., 2017; White et al., 2017; Zerbini, 2017).Por último, algunos autores hablan ya de una quinta etapa, que prestaría atención a los sistemas interconectados, sin necesidad de la intervención humana en muchos casos, el internet de las cosas, con una presencia fundamental de las nuevas tecnologías y el análisis de los datos y de la información -más si cabe todavía- a la hora de tomar decisiones de marketing.

\subsection{El marketing internacional.}

A la hora de considerar el marketing internacional, muchos investigadores (Aulakh, 1997; Keegan, 2001; Czinkota y Ronkanien, 2013) han llegado a la conclusión de que los factores que afectan tanto al marketing nacional como al internacional comparten una base bastante parecida. Sin embargo, existen algunas cuestiones que se asocian únicamente al segundo, debido a la complejidad de un escenario en el que entran en juego distintos entornos con importantes diferencias sociales, económicas, políticas o legales. Así pues, la forma de abordar estos mercados internacionales ha sido una cuestión que ha recibido especial atención desde la década de los 60, con teorías que se centraban en la dicotomía entre llevar a cabo una inversión extranjera directa o apostar por la exportación. En los 70, el enfoque de la internacionalización identificaba la cesión de licencias, las franquicias o la subcontratación como decisiones estratégicas a la hora de abrir nuevos mercados. Ya en los 90, otros autores (Root, 1994; Buckley y Cason, 1998; Pan y Tse, 2000) comenzaron a analizar la inversión en economías emergentes con altos costes e importantes diferencias en el entorno, introduciendo alternativas como las jointventures o los contratos específicos. Y se identificaron como principales variables para la inversión en mercados extranjeros los costes de localización, los factores de financiación, la distancia cultural, la estructura del mercado o la estrategia competitiva (Buckley y Casson, 1998).

También el marco teórico sobre el que se asientan los numerosos análisis empíricos llevados a cabo en el campo de la internacionalización nos lleva por distintos caminos. El enfoque del modelo de Uppsala (Johanson y Vahlne, 1977, 2009) determina que el proceso de internacionalización de las empresas es gradual y que la estrategia de marketing en mercados internacionales se basa en la experiencia previa desarrollada por cada empresa, bien en el mercado de origen o en otros mercados internacionales. No obstante, es el enfoque basado en los recursos de la empresa (ya descrito anteriormente) el que ha contribuido en mayor medida a la determinación de los factores que influyen en el resultado de las empresas exportadoras (Zou y Stan, 1998; Leonidou et al., 2002; Sousa et al., 2008; Chugan y Singh, 2014; Chen et al., 2016), situando la estrategia de marketing internacional en el centro de la mayor parte de modelos empíricos (ver tabla 2). Del mismo modo, el enfoque de las capacidades dinámicas destaca la importancia de la estrategia de marketing en los mercados internacionales (derivado de un análisis de fuera hacia adentro), sin olvidar la aportación dela tecnología (análisis de dentro hacia afuera) en el desarrollo de nuevas capacidades (Asseraf y Shoham, 2014). Por otra parte, el enfoque basado en las instituciones (IBV) presta una mayor atención a las instituciones debido al apoyo que prestan a las empresas exportadoras y a la influencia que 
esto supone sobre sus decisiones de gestión estratégica (Dacin et al., 2002; Peng et al., 2008; Lipuma et al., 2013; Chenet al., 2016). La teoría de la contingencia, por su parte, destaca que una adecuada política de internacionalización implica una combinación de factores internos y externos (Harrigan, 1983; Hultman et al., 2011; Chen et al., 2016). Y la teoría del aprendizaje en la organización establece la relación entre las operaciones anteriores de la empresa y el resultado futuro (Lages et al., 2008; Santos-Vijande et al., 2012; Wei et al., 2014). La existencia de este gran número de enfoques teóricos, muchas veces relacionados entre sí, nos ofrece una idea de la falta de uniformidad y de la imposibilidad de considerar un solo punto de vista en el análisis de las políticas de marketing e internacionalización.

\begin{tabular}{|c|c|}
\hline \multicolumn{2}{|c|}{ TABLA 2. FACTORES PARA LA INTERNACIONALIZACIÓN } \\
\hline VARIABLES INTERNAS & VARIABLES EXTERNAS \\
\hline $\begin{array}{l}\text { ESTRATEGIA MARKETING INTERNAC. } \\
\text { Producto } \\
\text { Precio } \\
\text { Promoción } \\
\text { Distribución } \\
\text { Decisiones de Marketing Estratégico }\end{array}$ & $\begin{array}{l}\text { CARACT. MERCADOS INTERNAC. } \\
\text { Existencia de barreras } \\
\text { Entorno social, cultural, político, legal... }\end{array}$ \\
\hline $\begin{array}{l}\text { CARACTERÍSTICAS DE LA EMPRESA } \\
\text { Tamaño/Años experiencia } \\
\text { Innovación tecnológica } \\
\text { Experiencia internacional } \\
\text { Localización } \\
\text { Recursos /capacidades de la empresa }\end{array}$ & $\begin{array}{l}\text { CARACT. MERCADO NACIONAL } \\
\text { Apoyo a la exportación } \\
\text { Existencia de barreras } \\
\text { Entorno legal y político }\end{array}$ \\
\hline $\begin{array}{l}\text { CARACTERÍSTICAS DE LA DIRECCIÓN } \\
\text { Experiencia en exportación } \\
\text { Compromiso y actitud hacia la exportación } \\
\text { Análisis de los mercados internacionales }\end{array}$ & \\
\hline
\end{tabular}

Fuente: Adaptado de Chugan y Singh, 2014; Chen et al., 2016.

Por último, desde el punto de vista operativo cabe destacar dos importantes enfoques en lo que a estrategia de marketing internacional se refiere: (1) las políticas de estandarización vs adaptación, en las que las empresas tienden a desarrollar programas estándar de marketing para los distintos países extranjeros con el fin de reducir sus costes mediante economías de escala (estandarización), o se ven obligadas a adaptar sus políticas a los distintos mercados (adaptación) cuando la primera opción no es posible, y (2) las políticas de concentración vs diversificación, donde las empresas tienden a concentrar sus esfuerzos en pocos mercados, normalmente los más atractivos (concentración), o bien se centran en un gran número de mercados (diversificación). Como ya avanzaba la teoría de las capacidades dinámicas, no existe una única estrategia válida para todas las empresas, sino que la elección de una u otra opción dependerá de cuestiones como el tipo de negocio o las características de los mercados. En este sentido, autores como Zou y Cavusgil (2002), defienden la existencia de diferentes estrategias internacionales, intentando integrar todas las posibles alternativas en un modelo de estrategia global de marketing, al contrario de lo que ocurre con los mercados nacionales donde solo existe una única estrategia. 


\section{METODOLOGÍA}

El análisis teórico de las disciplinas del marketing y la internacionalización han proporcionado la base necesaria para el desarrollo de numerosas investigaciones con un trasfondo empírico que pretenden demostrar la relación entre las principales variables que afectan al resultado exportador (ver tabla 2) en distintos sectores y/o mercados. En todos ellos la estrategia de marketing internacional juega un papel fundamental en la determinación del rendimiento final de la empresa, y es a su vez condicionado por una serie de inputs como el entorno, el management o los recursos de la empresa (Cavusgil y Zou, 1994; Zou y Stan, 1998; Katsikeas et al., 2000, 2006; Chugan y Singh 2014, Chen et al., 2016). Es por ello que esta investigación pretende profundizar en dicha estrategia y la forma en la que se lleva a cabo, ya que juega un papel fundamental en el sector de la moda. Además, en el caso que nos ocupa, el análisis de la estrategia de marketing internacional considerará dos aspectos diferenciados: (1) el enfoque estratégico y (2) el enfoque operativo. A su vez, se prestará una especial atención a la forma en que las nuevas tecnologías son incorporadas a las decisiones de marketing.

Para el análisis de los resultados, se partirá de las siguientes hipótesis que tienen por objetivo mostrar el grado de incorporación de las nuevas tendencias de marketing a las políticas de marketing de las empresas exportadoras que se van a analizar:

$\mathrm{H}_{1}$ : Las empresas del sector incorporan técnicas de marketing relacional a sus políticas estratégicas de marketing internacional.

$\mathrm{H}_{2}$ : Las empresas del sector incorporan técnicas de marketing relacional a sus políticas operativas de marketing internacional.

$\mathrm{H}_{3}$ : Las empresas del sector comienzan a incorporar las nuevas tecnologías a sus políticas estratégicas y operativas de marketing internacional.

Para ello, nuestra investigación, basada en técnicas cuantitativas, consistió en la entrega de un cuestionario a una muestra representativa de empresas exportadoras del sector de la moda hogar. Para la cuantificación del mismo, se utilizó la base de datos ORBIS, determinando la existencia de aproximadamente 680 empresas internacionalizadas que operan en dicha industria. Este artículo pretende mostrar las peculiaridades de este microsector mediante el análisis descriptivo de los ítems considerados en una muestra final de 35 empresas exportadoras españolas, seleccionadas por su trayectoria de internacionalización, la diversificación de los mercados y la regularidad de sus exportaciones. Para ello se utilizó un formulario de Google que fue enviado por correo electrónico directamente a los directivos de cada una de las empresas y un posterior contacto telefónico. Los resultados, obtenidos en 2017-18, se plasmaron sobre una escala Likert de 7 puntos que recogía las apreciaciones personales de cada directivo, si bien los resultados en este artículo se muestran en forma de escala decimal, mucho más fácil de interpretar. Los cálculos numéricos fueron realizados con el programa IBM SPSS 20 de tratamiento estadístico.

En lo que a escalas para el análisis estratégico se refiere, se adoptó el enfoque de las capacidades dinámicas y partimos de la siguiente literatura: Conant et al., 1990; Leonidou et al., 2002; Desarbo et al., 2005; Song et al., 2008; Murray et al., 2011; Morgan y Katsikeas, 2012 y Parnell et al., 2015. Para la definición del cuestionario en la parte operativa referente a las políticas de marketing mix, fueron determinantes los estudios de Leonidou et al. (2002) y Morgan et al. (2012), y la investigación de Prasad et al. (2001) para el caso de la incorporación de las nuevas tecnologías. 


\section{RESULTADOS}

Una vez definida la bibliografía que ha servido de base para la elaboración del cuestionario con los tres bloques principales que van a ser analizados (estrategia de marketing, marketing operativo y uso de las nuevas tecnologías), mostraremos los resultados obtenidos, siguiendo la misma estructura que se ha definido hasta el momento. Se incluyen las principales conclusiones de la literatura empírica y su comparación con los resultados obtenidos en el caso particular que estamos analizando.

\subsection{El marketing estratégico.}

El concepto de marketing estratégico se asocia a un enfoque basado en los recursos de la empresa (RBV) y se centra en cuestiones como la habilidad para segmentar y determinar mercados objetivos (targeting), el buen conocimiento de los clientes y competidores o la posibilidad de desarrollar relaciones en otros mercados, que en todos los casos presentan una relación positiva con el rendimiento empresarial (Leonidou et al., 2002). Para ello será fundamental la actitud e implicación de la alta dirección, debiendo aportar flexibilidad y apoyo a las políticas de exportación (Ibeh, 2003; Okpara, 2009; Cadogan et al., 2012; Chugan y Singh, 2014). También las capacidades desarrolladas por la empresa y la forma en que son integradas en la estrategia de marketing serán fundamentales para alcanzar una mayor ventaja competitiva (Srivastava et al., 1999; Vorhies y Morgan; 2005; Ramaswami et al., 2009; Morgan et al., 2012). Otros autores (Zou y Stan, 1998; Sousa et al., 2008) determinaron que la planificación de la estrategia exportadora y la investigación de mercados juegan un papel determinante a la hora de mejorar el resultado empresarial. En lo que se refiere a otros activos intangibles ligados al marketing relacional, cuestiones como la orientación a los mercados internacionales, las relaciones con socios estratégicos y proveedores o las capacidades para relacionarse con el mercado también presentan un efecto positivo en el rendimiento final de la empresa (Ibeh, 2003; Lages et al., 2005, 2009; Karelakis et al., 2008; Sousa et al., 2008). Por otra parte, destacar que un paso más en las relaciones con el mercado lo constituyen el nivel de responsabilidad social corporativa y las estrategias orientadas al cuidado medioambiental desarrolladas por las empresas, y han demostrado tener también un efecto positivo sobre el beneficio empresarial (Antonietti y Marzucchi, 2014; Chugan y Singh, 2014; Zeriti et al., 2014).

En el caso que nos ocupa, si analizamos el tipo de estrategia competitiva que desarrollan las empresas citadas anteriormente en mercados internacionales, observamos que casi una tercera parte de ellas se centran en aspectos directamente relacionados con el marketing (bien a través de aplicaciones de nuevas técnicas, del control sobre la distribución, de la implantación de una política de precios competitiva, o mediante una adecuada segmentación del mercado), en torno al 60 por cien basan su estrategia en la innovación y el desarrollo de nuevos productos, y una tasa superior al 10 por cien no tiene una política definida.

Además, cuando fueron preguntados por la estrategia de marketing, los directivos encuestados afirmaron, con valores próximos a 8 (sobre 10), estar mejor preparados que la competencia en el conocimiento de clientes y competidores, en la efectividad del equipo de ventas y del servicio post-venta, y en la habilidad para retener clientes y desarrollar políticas de Responsabilidad Social Corporativa, cuestiones que vienen a destacar la gran influencia de los nuevos enfoques de marketing relacional en el planteamiento estratégico de las políticas de marketing internacional. Presentaron valoraciones más bajas, pero todavía positivas (por encima de 6), aspectos como la habilidad para segmentar y seleccionar mercados, el desarrollo de programas de investigación de mercados y la planificación de las actividades de marketing, aspectos que, sin duda, pueden ser mejorados (ver tabla 3). 


\section{TABLA 3. ÍTEMS Y VALORACIONES DE LAS POLÍTICAS DE MARKETING ESTRATÉGICO}

\begin{tabular}{|c|c|c|}
\hline ÍTEMS MARKETING ESTRATÉGICO & $\begin{array}{l}\text { Valores } \\
\text { medios } \\
\end{array}$ & $\begin{array}{c}\text { Desviación } \\
\text { estándar }\end{array}$ \\
\hline \multicolumn{3}{|l|}{ MARKETING ESTRATÉGICO } \\
\hline MS1: Conocimiento de los clientes & 7,63 & 0,92 \\
\hline MS2: Conocimiento de la competencia & 7,51 & 0,69 \\
\hline MS3: Planificación de las actividades de marketing & 6,90 & 0,97 \\
\hline MS4: Integración de las actividades de marketing & 6,08 & 1,05 \\
\hline MS5: Habilidad para segmentar y reconocer mercados & 6,94 & 0,87 \\
\hline MS6: Desarrollo de proyectos de investigación de mercados & 6,78 & 1,25 \\
\hline MS7: Relación con los miembros del canal & 7,35 & 0,96 \\
\hline MS8: Cuidado de la imagen corporativa & 7,47 & 1,02 \\
\hline MS9: Habilidad para retener a los clientes & 7,76 & 0,73 \\
\hline MS10:Efectividad del servicio post-venta & 7,67 & 0,76 \\
\hline MS11: Efectividad del equipo comercial & 7,63 & 0,86 \\
\hline
\end{tabular}

Fuente: elaboración propia.

Estas respuestas, por tanto, no solo vienen a demostrar que se acepta la primera de las hipótesis definidas, sino que nos muestran cuáles son las fortalezas y debilidades a la hora de definir las características del marketing estratégico.

\subsection{El marketing operativo.}

El marketing operativo ha consistido tradicionalmente en la implementación de las políticas de marketing mix (producto, precio, promoción y distribución), a las que se han ido añadiendo nuevas variables relacionadas con los enfoques más vanguardistas, fundamentalmente el marketing relacional y las nuevas tecnologías. Uno de los temas que mayor repercusión ha tenido es el grado de adaptación o estandarización de los componentes de la estrategia de marketing mix, mostrando distintos resultados en lo que a efecto sobre el resultado empresarial se refiere (Chugan y Singh, 2014; Chen et al., 2016). Esto es totalmente consistente con las teorías de la contingencia y de las capacidades dinámicas ya que no solo es importante la política de marketing mix en sí, sino la forma en que se implementa y se lleva a cabo la estrategia, que es distinta en cada empresa (Cavusgil y Zou, 1994; Zou y Stan, 1998; Katsikeas et al., 2006; Sousa et al., 2008; Morgan et al., 2012; Chen et al., 2016).

Si analizamos cada una de las variables del marketing mix, podemos obtener una relación de aspectos a considerar en cada caso y el efecto sobre los resultados empresariales. En lo que a producto se refiere, aspectos como la originalidad del producto o la habilidad para desarrollar nuevos productos de aceptación en el mercado potencian las capacidades de la empresa y tienen un efecto positivo en la empresa (O'Cass y Julian, 2003; Boehe y Cruz, 2010; Gashi et al., 2014). Si hablamos de precio cobran importancia factores como la forma de fijar los precios, la adopción de políticas de precios de prestigio o de penetración, la política de crédito, la estrategia con respecto a la divisa y el ajuste de precios a las condiciones del mercado (Chetty y Hamilton, 1993; Zou y Stan, 1998; Karelakis et al., 2008). Con respecto a la política de promoción, son factores determinantes la publicidad, la venta directa o las ferias internacionales, que contribuyen a mejorar el resultado (Leonidou et al., 
2002; Morgan et al., 2012) y cobra una especial importancia el marketing relacional, al igual que en el caso de la distribución, resultando de importancia en este último caso aspectos como la motivación y el apoyo a socios y distribuidores (Ibeh, 2003; Lages et al., 2005, 2009; Karelakis et al., 2008).

En el caso del sector que nos ocupa, al analizar la estrategia de marketing mix, los resultados arrojan valores muy positivos para la política de producto (todos los ítems entre 7,5 y 8,2 en una escala sobre 10), destacando la inversión en calidad, diseño e innovación del producto, así como en marca y en la presentación de una amplia gama, además de adaptar el producto a las necesidades del cliente e intentar diferenciarse de la competencia. En lo que respecta al precio, las valoraciones que hacen los propios encuestados presentan ahora valores inferiores (entre 4,9 y 7,4) destacando la adaptación de la política de precios a los mercados internacionales, y la intención de mantener márgenes lo más elevados posible, por lo que las promociones basadas en descuentos y reducciones de precio presentan los valores más bajos. Respecto a la promoción, encontramos valores muy similares a los asociados a la política de precios, subrayando ahora la importancia de la adaptación de la comunicación a los mercados internacionales, la asistencia a ferias, las visitas a clientes y el uso del idioma del país de destino, y unos resultados muy mejorables en lo que al uso de redes sociales se refiere y a la promoción a través de medios publicitarios. Finalmente, en relación con la distribución, obtenemos valores muy dispares y destacan la adaptación de las políticas a los mercados internacionales, el uso de agentes y distribuidores y el apoyo a los miembros del canal. Las empresas, sin embargo, no suelen recurrir a la figura de las filiales comerciales o de producción en el extranjero, en torno al 60 por cien vende directamente a los clientes extranjeros, y aproximadamente la mitad vende a través de internet, cifras que también presentan un alto grado de mejora (ver tabla 4).

\section{TABLA 4. ÍTEMS Y VALORACIONES DE LAS POLÍTICAS DE MARKETING MIX}

\begin{tabular}{|c|c|c|}
\hline ÍTEMS POLÍTICAS DE MARKETING OPERATIVO & $\begin{array}{l}\text { Valores } \\
\text { medios }\end{array}$ & $\begin{array}{c}\text { Desviación } \\
\text { estándar }\end{array}$ \\
\hline \multicolumn{3}{|l|}{ PRODUCTO } \\
\hline PD1: Adaptamos el producto a las necesidades de los clientes en otros mercados & 7,47 & 0,90 \\
\hline PD2: Ofrecemos una amplia gama de productos & 7,67 & 0,96 \\
\hline PD3: Invertimos en la calidad del producto & 8,16 & 0,78 \\
\hline PD4: Invertimos en potenciar la marca en mercados internacionales & 7,47 & 1,04 \\
\hline PD5: Invertimos en el diseño del producto internacional & 7,67 & 1,12 \\
\hline PD6: Invertimos en la innovación del producto & 7,51 & 1,20 \\
\hline PD7: Ofrecemos un excelente servicio al cliente & 7,76 & 0,73 \\
\hline PD8:Diferenciamos el producto de la competencia internacional & 8,04 & 1,04 \\
\hline \multicolumn{3}{|l|}{ PRECIO } \\
\hline PC1: Adaptamos la estrategia de precios a los mercados exteriores & 7,39 & 0,88 \\
\hline PC2: Seguimos una estrategia de precios altos & 6,20 & 1,41 \\
\hline PC3: Ofrecemos precios muy competitivos & 5,92 & 1,29 \\
\hline PC4: Mantenemos un alto margen comercial & 5,92 & 1,27 \\
\hline PC5: Controlamos el precio final en los mercados exteriores & 5,84 & 1,11 \\
\hline PC6: Fijamos los precios según los costes del producto & 6,65 & 0,92 \\
\hline PC7: Realizamos descuentos y promociones basados en el precio con frecuencia & 4,94 & 1,00 \\
\hline PC8: Proporcionamos crédito a nuestros clientes internacionales & 6,04 & 1,29 \\
\hline
\end{tabular}


González-Ferriz, F., Sánchez-García, J., Garrigos-Simon, F. J.

\begin{tabular}{|c|c|c|}
\hline \multicolumn{2}{|l|}{ PROMOCIÓN } & \multirow[b]{2}{*}{0,93} \\
\hline PR1: Adaptamos la política de promoción a los mercados internacionales & 7,14 & \\
\hline PR2: Acudimos a ferias internacionales & 7,47 & 1,35 \\
\hline PR3: Visitamos a nuestros clientes en el extranjero con frecuencia & 6,78 & 1,13 \\
\hline PR4: Hacemos publicidad en medios de comunicación internacionales & 5,27 & 1,67 \\
\hline PR5: Disponemos de catálogos propios adaptados según los mercados & 7,14 & 1,55 \\
\hline PR6: Realizamos mailings con frecuencia a nuestros clientes de internacional & 5,88 & 1,14 \\
\hline PR7: Adaptamos la web a los mercados exteriores & 6,78 & 1,29 \\
\hline PR8: Utilizamos redes sociales en otros países & 4,78 & 1,49 \\
\hline PR9: Utilizamos el idioma del mercado al que nos dirigimos & 7,80 & 1,18 \\
\hline \multicolumn{3}{|l|}{ DISTRIBUCIÓN } \\
\hline PL1: Adaptamos nuestra política de distribución a los mercados internacionales & 7,67 & 0,90 \\
\hline PL2: Ofrecemos apoyo al canal elegido en cada mercado & 7,43 & 0,95 \\
\hline PL3: Utilizamos agentes internacionales & 7,76 & 0,90 \\
\hline PL4: Utilizamos distribuidores & 7,06 & 1,43 \\
\hline PL5: Tenemos filiales comerciales & 3,59 & 1,98 \\
\hline PL6: Tenemos filiales de producción & 2,73 & 1,63 \\
\hline PL7: Vendemos directamente al cliente internacional final & 4,73 & 1,69 \\
\hline PL8: Vendemos en otros países a través de Internet & 3,96 & 1,33 \\
\hline PL9: Cuidamos las fechas de entrega en los mercados internacionales & 8,08 & 0,75 \\
\hline
\end{tabular}

Fuente: elaboración propia.

Cobra especial atención el hecho de que nuestras empresas hacen un gran esfuerzo para potenciar la relación con el cliente a través del marketing operativo y conseguir de esa forma su fidelización, bien ofreciendo un excelente servicio en la mayoría de los casos, manteniendo el contacto mediante visitas y asistencia a ferias internacionales o apoyando a los miembros del canal de distribución con el fin de cuidar las fechas de entrega. Todo ello, nos lleva a concluir la aceptación de la segunda hipótesis, aunque algunos aspectos (venta a través de internet o relación a través de redes sociales) pueden ser mejorables.

\subsection{El uso de las nuevas tecnologías}

Mención especial merece la aplicación de las nuevas tecnologías basadas en Internet a las políticas de marketing estratégico y operativo, ya que contribuyen tanto a la mejora de la obtención de información, como a su tratamiento, y a la potenciación de la comunicación interna y externa de la compañía, resultando en una mejora del rendimiento empresarial (Prasad et al., 2001; Song et al., 2008; Mathews et al., 2016). Igualmente, el uso del comercio electrónico determina una relación positiva entre la estrategia de marketing internacional y el rendimiento final de la empresa (Karavdic y Gregory, 2005). A pesar de ello, son todavía pocos los estudios en relación a este nuevo enfoque y deja la puerta abierta a una nueva línea de investigación.

Por tanto, en lo referente a la aplicación de las nuevas tecnologías a las políticas de marketing internacional, encontramos nuevamente valores dispares (entre 6,1 y 8,5 sobre 10) pero todos ellos por encima de 5. Podemos concluir que Internet se aplica fundamentalmente a la promoción y a la comunicación con los clientes y con el personal de venta, pero puede ser mejorado el uso de la tecnología en la prospección de mercados y en la mejora de la 
comunicación interna, así como en la recogida de información sobre clientes, competidores y entorno (ver tabla 5).

TABLA 5. ÍTEMS Y VALORACIONES DE LA INCORPORACIÓN DE LAS NNTT
\begin{tabular}{|l|c|c|}
\hline \multicolumn{2}{|c|}{ AL MARKETING OPERATIVO } \\
ÍTEMS INCORPORACIÓN DE NNTT & $\begin{array}{c}\text { Valores } \\
\text { medios }\end{array}$ & $\begin{array}{c}\text { Desviación } \\
\text { estándar }\end{array}$ \\
\hline INCORPORACIÓN DE NUEVAS TECNOLOGÍAS & 7,67 & 0,96 \\
\hline NT1: Promoción y comunicación de nuevos productos/servicios & 8,45 & 0,55 \\
\hline NT2: Entrega de catálogos digitales/online a clientes & 8,29 & 0,71 \\
\hline NT3: Respuesta a consultas de clientes & 7,39 & 0,81 \\
\hline NT4: Acceso del personal de ventas a información sobre productos, precios ... & 7,22 & 0,95 \\
\hline NT5: Envío de información sobre pedidos por parte de clientes/vendedores & 6,69 & 1,19 \\
\hline NT6: Apoyo online a distribuidores/intermediarios & 6,65 & 0,86 \\
\hline NT7: Obtención de información sobre el mercado & 6,12 & 1,21 \\
\hline NT8: Uso de la información de la web a efectos de prospección y marketing & 6,20 & 0,92 \\
\hline NT9: Mejora de la comunicación en la dirección general y la gestión de equipos & \\
\hline
\end{tabular}

Fuente: elaboración propia.

Los resultados obtenidos nos llevan a aceptar solo de forma parcial la tercera hipótesis, ya que la incorporación de nuevas tecnologías presenta todavía muchos puntos de mejora. Tal y como muestran los resultados de la tabla 4, las empresas suspenden en venta a través de internet en el extranjero o en la utilización de redes sociales en mercados exteriores, hecho que puede ser explicado en parte por el hecho de que muchas de ellas no venden directamente al cliente final. Además, los datos de la tabla 5 nos demuestran también que ciertos aspectos pueden ser mejorados en lo que a recogida y análisis de datos se refiere, y a comunicación a través de Internet.

\section{DISCUSIÓN Y CONCLUSIONES}

El concepto de internacionalización se asocia a un amplio campo de estudio que, desde el punto de vista de la literatura científica, ha acaparado gran interés desde hace décadas (Bilkey, 1978; Aaby y Slater, 1989; Zou y Stan, 1998; Chugan y Singh, 2014; Chen et al., 2016). Además, se compone de distintas disciplinas según el enfoque que queramos abarcar (gestión empresarial, gestión del conocimiento y del capital humano, implantación de políticas de marketing internacional, desarrollo de la tecnología...). Es por ello que, encontrar un único modelo que sea capaz de dar explicación a una determinada industria o mercado, es prácticamente imposible. En el caso de España, y más concretamente en el sector del diseño y la moda, las empresas se caracterizan por una constante adaptación de los productos a las tendencias del momento, debiendo lanzar al mercado nuevas colecciones cada temporada que cumplan las expectativas de sus clientes y satisfagan sus necesidades. Esto supone situar las políticas de marketing internacional en un lugar predominante dentro de la estrategia competitiva de la empresa, y por ello hemos considerado ofrecer la máxima prioridad a este activo intangible. Las empresas de este sector van a ser capaces de externalizar determinados aspectos de la gestión, incluso departamentos tan importantes como la inversión en I+D+i que en muchos casos puede ser llevado a cabo por universidades o institutos tecnológicos, pero en ningún caso van a delegar las funciones de marketing. 
Dicho esto, nuestro estudio comienza con el análisis teórico del concepto de marketing y su evolución desde una concepción basada en el producto hacia otra centrada en los servicios (Vargo y Lusch, 2004, 2016; Payne et al., 2008). Al contrario de lo que se pudiera pensar, esto no va a suponer la desaparición de las políticas tradicionales de marketing (fundamentalmente producto, precio, promoción y distribución), sino más bien la aparición de nuevos enfoques, como la orientación al mercado (Narver y Slater, 1990; Jaworski y Kohli, 1993), el marketing relacional (Berry, 1983; Gummesson, 2002; Payne y Frow, 2005) o la incorporación de nuevas tecnologías (Prasad et al., 2001; Song et al., 2008; Parnell et al., 2015; Mathews et al., 2016),que vienen a complementar al anterior. En la misma dirección, cuando analizamos el entorno internacional encontramos una serie de publicaciones (Zou y Stan, 1998; Leonidou et al., 2002; Chugan y Singh, 2014; Chen et al., 2016) que han dirigido sus esfuerzos a la identificación de los factores que condicionan el rendimiento empresarial (ver tabla 2), y nuevamente la estrategia de marketing se posiciona en el centro de todos los modelos. Por último, vienen a completar el estudio una serie de teorías sin las cuales sería imposible entender la evolución de la mayor parte de modelos económicos hasta el momento actual. Destacan la teoría basada en los recursos (Prahalad y Hamel, 1990; Barney, 1991; Nelson, 1991; Teece, Pisano y Shuen, 1997; Wernerfelt, 1984, 1995; Katsikeas et al., 2000) que confiere una gran importancia a los activos tangibles e intangibles como determinantes de la ventaja competitiva de cada empresa, y la teoría de las capacidades dinámicas (Grant, 1991; Barney, 1991; Eisendhardt et al., 2000; Ruzzier et al., 2006) que aporta dinamismo al modelo en el sentido de que esos activos van cambiando con el tiempo en función de la evolución del entorno. Este último enfoque, aporta incluso más complejidad al escenario, ya que va a resultar muy difícil ofrecer un modelo que perdure en el tiempo y sea capaz de explicar de forma definitiva la relación entre las variables que condicionan los procesos de internacionalización de las empresas.

Desde el punto de vista empírico, también encontramos infinidad de publicaciones de las que podemos obtener una serie de conclusiones comunes a muchas de ellas. Así pues, se destaca la importancia de los activos intangibles de la empresa, fundamentalmente los directivos (Bouncken et al., 2015; Fernandez y Alegre, 2015; Parnell et al., 2015), porque ellos son los responsables de analizar e interpretar el entorno con el fin de implantar la mejor estrategia de marketing, que se ha presentado en todos los casos como determinante del rendimiento empresarial.

En el caso español que analiza el sector del mueble y la decoración de hogar, tanto la estrategia de marketing estratégico como la de marketing operativo han comenzado a incorporar los nuevos enfoques de marketing relacional, fundamentalmente a través de campañas de fidelización de clientes y de soporte a clientes, colaboradores y miembros del canal de distribución. Por su parte, las nuevas tecnologías están contribuyendo a mejorar la comunicación con el cliente, pero todavía queda un largo camino por recorrer en lo que a recogida de información para la prospección de mercado se refiere o la venta a través de Internet.

La contribución de nuestro estudio a la literatura existente puede analizarse desde dos puntos de vista. Por un lado pretende realizar un exhaustivo análisis de la disciplina del marketing internacional y su evolución a lo largo de las últimas décadas con el fin de definir unas escalas para el análisis cuantitativo soportadas por el entorno académico. Y por otro, ofrece a los lectores la posibilidad de conocer de primera mano la forma en la que un sector muy concreto de la economía española (la moda hogar) está adaptando sus políticas de marketing estratégico y operativo a la nueva realidad, mostrando tanto las fortalezas como las debilidades de esos procesos de adaptación. 
No obstante, somos conscientes de que, a pesar del incremento de los estudios provenientes de nuevas zonas económicas emergentes (fundamentalmente Asia y África), de la mayor complejidad estadística de los análisis empíricos, y de la incorporación de nuevos enfoques de marketing, todavía queda un amplio camino que recorrer en lo que respecta a la aplicación de las nuevas tecnologías a las políticas de marketing operativo, en aspectos como la utilización de big data para la predicción de comportamientos futuros, desarrollo de campañas de marketing digital , analítica web, venta por internet

\section{BIBLIOGRAFÍA}

Aaby, N. E., y Slater, S. F. (1989). Management influences on export performance: a review of the empirical literature 1978-88. International marketing review, vol. 6, $\mathrm{n}^{\circ}$ 4, pp. 726.

Ali, W., Frynas, J. G., y Mahmood, Z. (2017). Determinants of corporate social responsibility (CSR) disclosure in developed and developing countries: A literature review. Corporate Social Responsibility and Environmental Management, vol. 24, $\mathrm{n}^{\circ}$ 4, pp. 273-294.

Antonietti, R., y Marzucchi, A. (2014). Green tangible investment strategies and export performance: A firm-level investigation. Ecological Economics, n 108, pp. 150-161.

Asseraf, Y., y Shoham, A. (2014). The impact of strategic orientations on export marketing strategy: new classification and typology. In Research handbook on export marketing. Edward Elgar Publishing.

Atilgan, E., Aksoy, Ş., y Akinci, S. (2005). Determinants of the brand equity. Marketing intelligence y planning.

Aulakh, P. S. (1997). Global Marketing: Foreign Entry, Local Marketing, and Global Management.

Balodi, K. C. (2014). Strategic orientation and organizational forms: an integrative framework. European Business Review.

Barney, J. (1991). Firm resources and sustained competitive advantage. Journal of management, vol. 17, $\mathrm{n}^{\mathrm{0}}$ 1, pp. 99-120.

Barney, J. B., y Hesterly, W. S. (2015). Strategic management and competitive advantage: Cases and concepts.

Berry, L. L. (1983). Relationship marketing. Emerging perspectives on services marketing, vol. 66, $n^{\circ}$ 3, pp. 33-47.

Bilkey, W. J. (1978). An attempted integration of the literature on the export behavior of firms. Journal of international Business studies, vol. 9, $\mathrm{n}^{\circ}$ 1, pp. 33-46.

Boehe, D. M., y Cruz, L. B. (2010). Corporate social responsibility, product differentiation strategy and export performance. Journal of Business ethics, vol. 91, n 2, pp. 325346.

Bouncken, R. B., Schuessler, F., y Kraus, S. (2015). The theoretical embedding of born globals: Challenging existing internationalization theories. International Business $y$ Economics Research Journal (IBER), vol. 14, n 1, pp. 39-46.

Brakus, J. J., Schmitt, B. H., y Zarantonello, L. (2009). Brand experience: what is it? How is it measured? Does it affect loyalty? Journal of marketing, vol. 73, $n^{\circ} 3$, pp. 52-68.

Buckley, P. J., y Casson, M. C. (1998). Analyzing foreign market entry strategies: Extending the internalization approach. Journal of international business studies, vol. 29, $\mathrm{n}^{0} 3$, pp. 539-561.

Cadogan, J. W., Sundqvist, S., Puumalainen, K., y Salminen, R. T. (2012). Strategic flexibilities and export performance: The moderating roles of export market-oriented 
behavior and the export environment. European Journal of Marketing, vol. 46, $\mathrm{n}^{\mathrm{o}} 10$, pp. 1418-1452.

Cavusgil, S. T., y Zou, S. (1994). Marketing strategy-performance relationship: an investigation of the empirical link in export market ventures. Journal of marketing, vol. 58, $\mathrm{n}^{\mathrm{0}} 1$, pp. 1-21.

Chad, P. (2014). Organizational change within charities: improved performance via introduction of market orientation and other strategic orientations. International Review on Public and Nonprofit Marketing, vol. 11, no 1, pp. 89-113.

Chen, J., Sousa, C. M., y He, X. (2016). The determinants of export performance: a review of the literature 2006-2014. International Marketing Review.

Chetty, S. K., y Hamilton, R. T. (1993). Firm-level determinants of export performance: a meta-analysis. International Marketing Review.

Chugan, P. K., y Singh, S. (2014). Taxonomy for firm-level determinants of export performance. Universal Journal of Industrial and Business Management, vol. 2, $\mathrm{n}^{0} 1$, pp. 6-12.

Conant, J. S., Mokwa, M. P., y Varadarajan, P. R. (1990). Strategic types, distinctive marketing competencies and organizational performance: a multiple measures-based study. Strategic management journal, vol. 11, $\mathrm{n}^{\circ}$ 5, pp. 365-383.

Conner, K. R. (1991). A historical comparison of resource-based theory and five schools of thought within industrial organization economics: do we have a new theory of the firm?. Journal of management, vol. 17, nº 1, pp. 121-154.

Constantinides, E. (2014). Foundations of social media marketing. Procedia-Social and behavioral sciences, $\mathrm{n}^{\mathrm{0}} 148$, pp. 40-57.

Czinkota, M., y Ronkainen, I. (Eds.). (2011). The future of global business: a reader. Routledge.

Czinkota, M. R., y Ronkainen, I. A. (2013). International marketing. Cengage Learning.

Dacin, M. T., Goodstein, J., y Scott, W. R. (2002). INSTITUTIONAL THEORY AND INSTITUTIONAL CHANGE: INTRODUCTION TO THE SPECIAL RESEARCH FORUM. Academy of Management Journal, 45(1), 45-57.

Day, G. S. (1994). The capabilities of market-driven organizations. Journal of marketing, vol. 58, no 4, pp. 37-52.

DeSarbo, W. S., Anthony Di Benedetto, C., Song, M., y Sinha, I. (2005). Revisiting the Miles and Snow strategic framework: uncovering interrelationships between strategic types, capabilities, environmental uncertainty, and firm performance. Strategic management journal, vol. 26, n⿳⺈ 1, pp. 47-74.

Devece, C., Llopis-Albert, C., y Palacios-Marqués, D. (2017). Market orientation, organizational performance, and the mediating role of crowdsourcing in knowledge-based firms. Psychology y Marketing, vol. 34, n 12, pp. 1127-1134.

Eisenhardt, K. M., y Martin, J. A. (2000). Dynamic capabilities: what are they? Strategic management journal, vol. 21, $\mathrm{n}^{\mathrm{o}}$ 10-11, pp. 1105-1121.

El-Gohary, H. (2010). E-Marketing-A literature Review from a Small Businesses perspective. International journal of business and social science, vol. $1, \mathrm{n}^{\circ} 1$.

Fahey, L. (1999). Competitors: outwitting, outmaneuvering, and outperforming. University of Texas Press.

Fernández-Mesa, A., y Alegre, J. (2015). Entrepreneurial orientation and export intensity: Examining the interplay of organizational learning and innovation. International Business Review, vol. 24, $\mathrm{n}^{\circ}$ 1, pp. 148-156.

Friedman, M. (2007). The social responsibility of business is to increase its profits. In Corporate ethics and corporate governance (pp. 173-178). Springer, Berlin, Heidelberg. 
Frow, P. E., y Payne, A. F. (2009). Customer relationship management: a strategic perspective. Journal of business market management, vol. 3, $\mathrm{n}^{0}$ 1, pp. 7-27.

Gashi, P., Hashi, I., y Pugh, G. (2014). Export behaviour of SMEs in transition countries. Small Business Economics, vol. 42, n 2, pp. 407-435.

Grant, R. M. (1991). The resource-based theory of competitive advantage: implications for strategy formulation. California management review, vol. 33, n 3, pp. 114-135.

Grönroos, C. (1990). Service Management and Marketing-Managing the Moments of Truth in Service Competition (Massachusetts, Maxwell MacMillan).

Gummesson, E. (1994a). Broadening and specifying relationship marketing. Asia-Australia Marketing Journal, vol. 2, no 1, pp. 31-43.

Gummesson, E. (1994b). Making relationship marketing operational. International Journal of service industry management.

Gummesson, E. (2002). Relationship marketing and a new economy: it's time for de-programming. Journal of services marketing.

Guo, C. (2002). Market orientation and business performance. European journal of marketing.

Haji-Basri, M. (2012). Marketing research contemporary themes and trends. Interdisciplinary Journal of Contemporary Research in Business, vol. 4. $n^{\circ}$ 5, pp. 17-24.

Harrigan, K. R. (1983). Research methodologies for contingency approaches to business strategy. Academy of Management Review, vol. 8, no 3, pp. 398-405.

Hultman, M., Katsikeas, C. S., y Robson, M. J. (2011). Export promotion strategy and performance: the role of international experience. Journal of international marketing, vol. 19, no 4, pp. 17-39.

Hunt, S. D., y Morgan, R. M. (1995). The comparative advantage theory of competition. Journal of marketing, vol. 59, $\mathrm{n}^{\circ}$ 2, pp. 1-15.

Hunt, S. D. (2000). A general theory of competition: too eclectic or not eclectic enough? Too incremental or not incremental enough? Too neoclassical or not neoclassical enough?. Journal of Macromarketing, vol. 20, $\mathrm{n}^{\mathrm{0}}$ 1, pp. 77-81.

Ibeh, K. I. (2003). On the internal drivers of export performance among Nigerian firms: empirical findings and implications. Management decision.

Jaworski, B. J., y Kohli, A. K. (1993). Market orientation: antecedents and consequences. Journal of marketing, vol. 57, $\mathrm{n}^{\circ} 3$, pp. 53-70.

Johanson, J., y Vahlne, J. E. (1977). The internationalization process of the firm-a model of knowledge development and increasing foreign market commitments. Journal of international business studies, vol. 8, $\mathrm{n}^{0}$ 1, pp. 23-32.

Johanson, J., y Vahlne, J. E. (2009). The Uppsala internationalization process model revisited: From liability of foreignness to liability of outsidership. Journal of international business studies, vol. 40, no 9, pp. 1411-1431.

Kaplan, A. M. (2012). If you love something, let it go mobile: Mobile marketing and mobile social media 4x4. Business horizons, vol. 55, n 2, pp. 129-139.

Karavdic, M., y Gregory, G. (2005). Integrating e-commerce into existing export marketing theories: A contingency model. Marketing Theory, vol. 5, nº 1, pp. 75-104.

Karelakis, C., Mattas, K., y Chryssochoidis, G. (2008). Greek wine firms: Determinants of export performance. Agribusiness: An International Journal, vol. 24, n 2, pp. 275297.

Katsikeas, C. S., Leonidou, L. C., y Morgan, N. A. (2000). Firm-level export performance assessment: review, evaluation, and development. Journal of the Academy of Marketing Science, vol. 28, nº 4, pp. 493-511. 
Katsikeas, C. S., Samiee, S., y Theodosiou, M. (2006). Strategy fit and performance consequences of international marketing standardization. Strategic management journal, vol. 27, nº 9, pp. 867-890.

Keegan, W. J., y Schlegelmilch, B. B. (2001). Global marketing management: A European perspective. Pearson Education.

Kohli, A. K., y Jaworski, B. J. (1990). Market orientation: the construct, research propositions, and managerial implications. Journal of marketing, vol. 54, $\mathrm{n}^{\circ} 2$, pp. 1 18.

Kohli, A. K., Jaworski, B. J., y Kumar, A. (1993). MARKOR: a measure of market orientation. Journal of Marketing research, vol. 30, $n^{\circ}$ 4, pp. 467-477.

Kotler, P., Kartajaya, H., y Setiawan, I. (2010). From products to customers to the human spirit; marketing 3.0. John Wiley y Sons Inc, vol. 2, $\mathrm{n}^{0} 8$.

Kotler, P., Kartajaya, H., y Setiawan, I. (2016). Marketing 4.0: Moving from traditional to digital. John Wiley y Sons.

Kozlenkova, I. V., Samaha, S. A., y Palmatier, R. W. (2014). Resource-based theory in marketing. Journal of the Academy of Marketing Science, vol. 42, nº 1, pp. 1-21.

Lages, C., Lages, C. R., y Lages, L. F. (2005). The RELQUAL scale: a measure of relationship quality in export market ventures. Journal of business research, vol. 58, $n^{\circ}$ 8, pp. 1040-1048.

Lages, L. F., Jap, S. D., y Griffith, D. A. (2008). The role of past performance in export ventures: a short-term reactive approach. Journal of International Business Studies, vol. 39, n 2, pp. 304-325.

Lages, L. F., Silva, G., y Styles, C. (2009). Relationship capabilities, quality, and innovation as determinants of export performance. Journal of international Marketing, vol. 17, $\mathrm{n}^{\circ}$ 4, pp. 47-70.

Lehmann, D. R. (2005). Journal evolution and the development of marketing. Journal of Public Policy y Marketing, vol. 24, n 1, pp. 137-142.

Leonidou, L. C., Katsikeas, C. S., y Samiee, S. (2002). Marketing strategy determinants of export performance: a meta-analysis. Journal of Business research, vol. 55, $\mathrm{n}^{0} 1$, pp. 51-67.

Line, N. D., y Runyan, R. C. (2012). Hospitality marketing research: Recent trends and future directions. International Journal of Hospitality Management, vol. 31, n 2, pp. 477488.

LiPuma, J. A., Newbert, S. L., y Doh, J. P. (2013). The effect of institutional quality on firm export performance in emerging economies: a contingency model of firm age and size. Small Business Economics, vol. 40, nº 4, pp. 817-841.

Mathews, S., Bianchi, C., Perks, K. J., Healy, M., y Wickramasekera, R. (2016). Internet marketing capabilities and international market growth. International Business Review, vol. 25, $\mathrm{n}^{\circ}$ 4, pp. 820-830.

Morgan, R. M., y Hunt, S. D. (1994). The commitment-trust theory of relationship marketing. Journal of marketing, vol. 58, $\mathrm{n}^{\circ} 3$, pp. 20-38.

Morgan, N. A., Katsikeas, C. S., y Vorhies, D. W. (2012). Export marketing strategy implementation, export marketing capabilities, and export venture performance. Journal of the academy of marketing science, vol. 40, n 2, pp. 271-289.

Murray, J. Y., Gao, G. Y., y Kotabe, M. (2011). Market orientation and performance of export ventures: the process through marketing capabilities and competitive advantages. Journal of the Academy of Marketing Science, vol. 39, n 2, pp. 252-269.

Narver, J. C., y Slater, S. F. (1990). The effect of a market orientation on business profitability. Journal of marketing, vol. 54, nº 4, pp. 20-35. 
Nelson, R. R. (1991). Why do firms differ, and how does it matter?. Strategic management journal, vol. 12, n ${ }^{\circ}$ S2, pp. 61-74.

Ngai, E. W. (2003). Internet marketing research (1987-2000): a literature review and classification. European journal of marketing.

O'Cass, A., y Julian, C. (2003). Examining firm and environmental influences on export marketing mix strategy and export performance of Australian exporters. European journal of marketing.

Oh, H., Kim, B. Y., y Shin, J. H. (2004). Hospitality and tourism marketing: recent developments in research and future directions. International Journal of Hospitality Management, vol. 23, $\mathrm{n}^{\circ}$ 5, pp. 425-447.

Okpara, J. O. (2009). Strategic choices, export orientation and export performance of SMEs in Nigeria. Management Decision.

Orlitzky, M., Siegel, D. S., y Waldman, D. A. (2011). Strategic corporate social responsibility and environmental sustainability. Business y society, vol. 50, $\mathrm{n}^{0}$ 1, pp. 6-27.

Pan, Y., y Tse, D. K. (2000). The Hierarchical Model Entry Market Modes. Journal of International Business Studies, vol. 31, n 4, pp. 535-554.

Parnell, J. A., Long, Z., y Lester, D. (2015). Competitive strategy, capabilities and uncertainty in small and medium sized enterprises (SMEs) in China and the United States. Management Decision.

Payne, A., y Frow, P. (2005). A strategic framework for customer relationship management. Journal of marketing, vol. 69, $\mathrm{n}^{\circ}$ 4, pp. 167-176.

Payne, A. F., Storbacka, K., y Frow, P. (2008). Managing the co-creation of value. Journal of the academy of marketing science, vol. 36, $\mathrm{n}^{\circ} 1$, pp. 83-96.

Payne, A., y Frow, P. (2017). Relationship marketing: looking backwards towards the future. Journal of services marketing.

Peña, A. I. P., Jamilena, D. M. F., y Molina, M. Á. R. (2012). Validation of a market orientation adoption scale in rural tourism enterprises. Relationship between the characteristics of the enterprise and extent of market orientation adoption. International Journal of Hospitality Management, vol. 31, $\mathrm{n}^{\circ}$ 1, pp. 139-151.

Peng, M. W., Wang, D. Y., y Jiang, Y. (2008). An institution-based view of international business strategy: A focus on emerging economies. Journal of international business studies, vol. 39, n 5, pp. 920-936.

Penrose, E., y Penrose, E. T. (2009). The Theory of the Growth of the Firm. Oxford university press.

Pine, B. J., Pine, J., y Gilmore, J. H. (1999). The experience economy: work is theatre y every business a stage. Harvard Business Press.

Prahalad, C. K., y Hamel, G. (1990). Core competency concept. Harvard Business Review, vol. 64, $\mathrm{n}^{\mathrm{O}}$ 3, pp. 70-92.

Prasad, V. K., Ramamurthy, K., y Naidu, G. M. (2001). The influence of Internet-marketing integration on marketing competencies and export performance. Journal of International Marketing, vol. 9, nº 4, pp. 82-110.

Qader, I. K. A., y Omar, A. B. (2013). The evolution of experiential marketing: Effects of brand experience among the millennial generation. International journal of academic research in business and social sciences, vol. 3, $\mathrm{n}^{\circ}$ 7, pp. 331.

Ramaswami, S. N., Srivastava, R. K., y Bhargava, M. (2009). Market-based capabilities and financial performance of firms: insights into marketing's contribution to firm value. Journal of the Academy of Marketing Science, vol. 37, n 2, pp. 97.

Ricci, L. A., y Trionfetti, F. (2012). Productivity, networks, and export performance: Evidence from a cross-country firm dataset. Review of International Economics, vol. 20, no 3, pp. 552-562. 
Root, F. R. (1994). Entry strategies for international markets. Jossey-Bass.

Ruzzier, M., Hisrich, R. D., y Antoncic, B. (2006). SME internationalization research: past, present, and future. Journal of Small Business and Enterprise Development, vol. 13, ${ }^{\circ}$ 4, pp. 476-497.

Saleh, M. A. H. (2016). Themes and topics of research in marketing: A content analysis of the articles published in three marketing journals. International Journal of Business Administration, vol. 7, $\mathrm{n}^{\circ}$ 1, pp. 12.

Santos-Vijande, M. L., López-Sánchez, J. Á., y Trespalacios, J. A. (2012). How organizational learning affects a firm's flexibility, competitive strategy, and performance. Journal of business research, vol. 65, nº 8, pp. 1079-1089.

Saravanakumar, M., y SuganthaLakshmi, T. (2012). Social media marketing. Life Science Journal, vol. 9, $\mathrm{n}^{\circ}$ 4, pp. 4444-4451.

Schmitt, B. (2000). H (1999) Experiential Marketing: How to Get Customers to Sense, Feel, Think, Act, and Relate to Your Company and Brands.

Schmitt, B. H., Brakus, J., y Zarantonello, L. (2014). The current state and future of brand experience. Journal of Brand Management, vol. 21, $\mathrm{n}^{\circ}$ 9, pp. 727-733.

Shafei, R., y Zohdi, M. (2014). Relational capabilities in market orientation to improvement of performance outcomes in SMEs. International Journal of Business Performance Management, vol. 15, $\mathrm{n}^{\circ}$ 4, pp. 295-315.

Song, M., Nason, R. W., y Di Benedetto, C. A. (2008). Distinctive marketing and information technology capabilities and strategic types: A cross-national investigation. Journal of International Marketing, vol. 16, $\mathrm{n}^{\mathrm{0}}$ 1, pp. 4-38.

Sousa, C. M., Martínez-López, F. J., y Coelho, F. (2008). The determinants of export performance: A review of the research in the literature between 1998 and 2005 . International Journal of Management Reviews, vol. 10, nº 4, pp. 343-374.

Srivastava, R. K., Shervani, T. A., y Fahey, L. (1999). Marketing, business processes, and shareholder value: an organizationally embedded view of marketing activities and the discipline of marketing. Journal of marketing, vol. 63, nº 4_suppl1, pp. 168-179.

Srivastava, R. K., Fahey, L., y Christensen, H. K. (2001). The resource-based view and marketing: The role of market-based assets in gaining competitive advantage. Journal of management, vol. 27, $\mathrm{n}^{\circ} 6$, pp. 777-802.

Teece, D. J., y Pisano, G. (1997). A. Shuen (1997), "Dynamic capabilities and strategic management”. Strategic management journal, vol. 18, n 7, pp. 509-533.

Vargo, S. L., y Lusch, R. F. (2004). Evolving to a new dominant logic for marketing. Journal of marketing, vol. 68, $\mathrm{n}^{\circ} 1$, pp. 1-17.

Vargo, S. L., y Lusch, R. F. (2016). Institutions and axioms: an extension and update of service-dominant logic. Journal of the Academy of marketing Science, vol. 44, $\mathrm{n}^{0} 1$, pp. 5-23.

Vivek, S. D., Beatty, S. E., y Morgan, R. M. (2012). Customer engagement: Exploring customer relationships beyond purchase. Journal of marketing theory and practice, vol. 20, no 2, pp. 122-146.

Vorhies, D. W., y Morgan, N. A. (2005). Benchmarking marketing capabilities for sustainable competitive advantage. Journal of marketing, vol. 69, $\mathrm{n}^{\circ}$ 1, pp. 80-94.

Wei, Y. S., Samiee, S., y Lee, R. P. (2014). The influence of organic organizational cultures, market responsiveness, and product strategy on firm performance in an emerging market. Journal of the Academy of Marketing Science, vol. 42, nº 1, pp. 49-70.

Wernerfelt, B. (1984). A resource-based view of the firm. Strategic management journal, vol. 5, $n^{\circ}$ 2, pp. 171-180.

Wernerfelt, B. (1995). The resource-based view of the firm: Ten years after. Strategic management journal, vol. 16, $\mathrm{n}^{\mathrm{0}} 3$, pp. 171-174. 
White, C. L., Nielsen, A. E., y Valentini, C. (2017). CSR research in the apparel industry: A quantitative and qualitative review of existing literature. Corporate social responsibility and environmental management, vol. 24, n 5, pp. 382-394.

Yadav, M. S. (2010). The decline of conceptual articles and implications for knowledge development. Journal of Marketing, vol. 74, $\mathrm{n}^{\circ}$ 1, pp. 1-19.

Zerbini, F. (2017). CSR initiatives as market signals: A review and research agenda. Journal of Business Ethics, vol. 146, no 1, pp. 1-23.

Zeriti, A., Robson, M. J., Spyropoulou, S., y Leonidou, C. N. (2014). Sustainable export marketing strategy fit and performance. Journal of International Marketing, vol. 22, $\mathrm{n}^{\mathrm{o}}$ 4, pp. 44-66.

Zou, S., y Stan, S. (1998). The determinants of export performance: a review of the empirical literature between 1987 and 1997. International marketing review.

Zou, S., y Cavusgil, S. T. (2002). The GMS: A broad conceptualization of global marketing strategy and its effect on firm performance. Journal of marketing, vol. 66, nº 4, pp. 40 